\title{
PyWRKY26 and PybHLH3 cotargeted the PyMYB114 promoter to regulate anthocyanin biosynthesis and transport in red-skinned pears
}

Chuang $\mathrm{Li}^{1}$, Jun Wu${ }^{2}$, Kang-Di Hu', Shu-Wei Wei ${ }^{3}$, Hong-Ye Sun ${ }^{1}$, Lan-Ying Hu ${ }^{1,4}$, Zhuo Han ${ }^{1}$, Gai-Fang Yao and Hua Zhang

\begin{abstract}
Red pear is favored because of its bright appearance and abundant anthocyanins. Anthocyanin biosynthesis is controlled by transcription factors (TFs) forming regulatory complexes. In red-skinned pears, the WRKY TFs have a significant relationship with anthocyanin biosynthesis, but the molecular mechanism of the WRKY TFs involved in regulating color formation in red-skinned pear is unclear. In this study, the TFs PyWRKY31 and PyWRKY26 were screened as candidate genes for controlling anthocyanin biosynthesis by transcriptome data and bioinformatics analysis. The effect of anthocyanin accumulations after cotransformation of PyWRKY31 or PyWRKY26 with its partners PyMYB10, PyMYB114, and PybHLH3 was verified in tobacco leaves and strawberry receptacles by a transient expression system. RT-qPCR analysis and a dual-luciferase reporter system further confirmed that this cotransformation activated the expression of PyDFR, PyANS, and PyUFGT in anthocyanin biosynthesis and PyGST in anthocyanin transport instead of the PYABC transporter and PyAVP. Furthermore, the cotransformed PyWRKY26 and PybHLH3 could bind to the PyMYB114 promoter, and PYWRKY26 directly activated the transcription of PyMYB114. In addition, the TF PyWRKY26 could interact with PybHLH3, as confirmed by firefly luciferase complementation and yeast two-hybrid $(\mathrm{Y} 2 \mathrm{H})$ assays. These results showed that the interaction of PyWRKY26 and PybHLH3 could cotarget the PyMYB114 promoter, which resulted in anthocyanin accumulation in red-skinned pear. This study further strengthened the understanding of the regulatory mechanism of anthocyanin accumulation and contributed to improving the appearance of red-skinned pears.
\end{abstract}

\section{Introduction}

Pear (Pyrus L.) is one of the most common and popular fruits in the world, and red pears are favored by consumers for their beautiful appearance and abundant anthocyanins ${ }^{1}$. In plant tissues, anthocyanins are widely present; these molecules are important flavonoids with multiple physiological functions that aid pollination, seed

\footnotetext{
Correspondence: Gai-Fang Yao (2017800495@hfut.edu.cn) or

Hua Zhang (hzhanglab@hfut.edu.cn)

'School of Food and Biological Engineering, Hefei University of Technology, 230009 Hefei, China

${ }^{2}$ Centre of Pear Engineering Technology Research, State Key Laboratory of Crop Genetics and Germplasm Enhancement, Nanjing Agricultural University, 210095 Nanjing, China

Full list of author information is available at the end of the article.

These authors contributed equally: Chuang Li, Jun Wu, Kang-Di Hu.
}

dispersal, and resistance to adverse environmental conditions $^{2}$. In addition, anthocyanins have significant antioxidant activity and potential benefits for human health, such as reducing the risk of cancer, inflammation, and coronary arteriosclerosis ${ }^{3-5}$.

Anthocyanin biosynthesis is coordinately regulated by structural genes and TFs in many species. The structural genes consist of seven enzyme genes, from phenylalanine ammonia-lyase (PAL) to UDP-glucose: flavonoid 3-Oglucosyltransferase (UFGT). In addition, synthetic anthocyanins are transported and stored in vacuoles. There are three mechanisms for anthocyanin transport: glutathione S-transferase (GST)-mediated transport, membrane transport, and vesicle trafficking ${ }^{6,7}$. GST can mobilize anthocyanins by acting as a carrier of these

\section{(c) The Author(s) 2020}

(c) (i) Open Access This article is licensed under a Creative Commons Attribution 4.0 International License, which permits use, sharing, adaptation, distribution and reproduction in any medium or format, as long as you give appropriate credit to the original author(s) and the source, provide a link to the Creative Commons license, and indicate if changes were made. The images or other third party material in this article are included in the article's Creative Commons license, unless indicated otherwise in a credit line to the material. If material is not included in the article's Creative Commons license and your intended use is not permitted by statutory regulation or exceeds the permitted use, you will need to obtain permission directly from the copyright holder. To view a copy of this license, visit http://creativecommons.org/licenses/by/4.0/. 
molecules, and anthocyanins are transported from the cytoplasm to the tonoplast through the $\mathrm{ABC}$ transmembrane transporter ${ }^{8,9}$. Many secondary transporters and channels, such as malate transporter and MATE-type transports and $\mathrm{ABC}$ transporters, were reported to exist on the tonoplast, and the activities of these transporters are directly or indirectly dependent on the proton gradient generated by the pyrophosphate-energized vacuolar membrane proton pump ${ }^{9-11}$.

By forming the MBW transcriptional complex, the MYB, bHLH, and WD40 proteins can regulate structural genes $^{12-14}$. MYBs, as central factors regulating anthocyanin biosynthesis, have been widely researched. In horticultural crops, apple $M d M Y B 10, M d M Y B 1$, and MdMYB110a; strawberry FaMYB10; bayberry MrMYB1; and pear $P y M Y B 10$, and $P y M Y B 114$ were reported to regulate anthocyanin biosynthesis in succession by forming the MBW transcriptional complex ${ }^{15-18}$. However, in different species, the regulatory mechanism of the MBW complex is different. In Arabidopsis and Myrica rubra, the three proteins in the MBW complex can interact with each other to form a transcriptional complex $^{19,20}$. In apple, MdMYB10 interacts with $M d b H L H 3$ and MdbHLH33 to enhance the synthesis of anthocyanins $^{15}$. In pears, PyMYB114 or PyMYB10 interacts with $P y b H L H 3$ to significantly enhance anthocyanin biosynthesis $^{18}$. Recently, overexpression of the SIMYB75 gene was shown to promote anthocyanin biosynthesis in tomato ${ }^{21}$.

In addition to the MBW complex, other TFs, such as NACs, ERFs, HY5, BBX22, and WRKY, were found to be involved in regulating anthocyanin biosynthesis ${ }^{22-24}$. These TFs could regulate anthocyanin biosynthesis by indirectly or directly binding to the MBW complex. The WRKY TFs belong to one large gene family that regulates a series of physiological processes, including development and senescence, and resistance to adverse environments ${ }^{24}$. WRKY TFs were identified by one or two conserved WRKY domain(s) usually followed by a zinc-finger motif. The WRKY TF contains a specific nucleic acid sequence $(\mathrm{C} / \mathrm{T}) \mathrm{TGAC}(\mathrm{T} / \mathrm{C})$ named the $\mathrm{W}$-box, which regulates the defense response to various stresses by self-regulation, and it could recognize and bind to the W-box or other promoters of the WRKY TFs to generate biological effects by achieving crosstalk of different WRKY ${ }^{25}$. Compared with the MYB TFs, WRKY is an emerging player in the plant signaling regulation network. The interaction between the upstream regulator of the WRKY TF and the downstream target gene constitutes a complex regulatory network ${ }^{25,26}$.

Recently, several reports have shown that WRKY proteins have an obvious correlation with the regulation of anthocyanin biosynthesis. For example, GbWRKY1 in Gossypium barbadense was proven to have a positive correlation with anthocyanin accumulation when expressed in Arabidopsis thaliana ${ }^{27}$. In a previous study, AtWRKY75 responded to low phosphate (Pi) stress by decreasing anthocyanin accumulation in $A$. thaliana seedlings ${ }^{28}$. Moreover, the Atwrky41 mutation resulted in increased anthocyanin content in $A$. thaliana rosette leaves ${ }^{29}$. AtWRKY6 promotes PR1 promoter activity, which is related to senescence and pathogen defense, and the plant responds to abiotic and biotic stresses by decreasing anthocyanin accumlation $^{30}$. Recently, it was reported that the WRKY TF PhPH3 in petunia correlates with changes in the color of petals by playing a role downstream of the MBW complex ${ }^{31}$. Amato et al. also proved that $V v W R K Y 26$, a homologous gene of $\mathrm{PhPH} 3$ in Vitis vinifera, induces the accumulation of flavonoids ${ }^{23}$. In apple, MdWRKY4O is a key modulator in wounding-induced anthocyanin biosynthesis ${ }^{32}$. Yang et al. reported that the WRKY family was related to anthocyanin biosynthesis in redskinned pear ${ }^{33}$. However, whether WRKYs are involved in anthocyanin biosynthesis by interacting with the TFs PyMYB114 and PybHLH3 in red pears is still unclear.

In this study, according to the transcriptome data of 'Starkrimson' and its green mutant pear at three fruit development stages, we screened two WRKY TFs and performed bioinformatics analysis. Furthermore, PyWRKY26 or PyWRKY31 with PyMYB114 and PybHLH3 were cotransformed into tobacco leaves and strawberry receptacles by a transient expression system. In addition, RT-qPCR analysis and dual-luciferase reporter system assays revealed the regulatory pattern of cotransformed PyWRKY26 with its partners to activate the activity of anthocyanin biosynthesis- and transport-related structural genes. Furthermore, firefly luciferase complementation and $\mathrm{Y} 2 \mathrm{H}$ assays confirmed the interaction of PyWRKY26 with PybHLH3. Our research reveals a potential mechanism of regulating anthocyanin biosynthesis in red-skinned pears, which will help elucidate the regulatory network to clarify anthocyanin accumulation in other species.

\section{Results}

Screening of the candidate WRKY genes by transcriptome data and bioinformatics analysis

A previous search by Yang et al. reported that the WRKY family was related to anthocyanin biosynthesis by RT-qPCR analysis in red-skinned pear ${ }^{33}$. To identify the function of WRKY genes in controlling anthocyanin biosynthesis in red pears, we screened 66 differentially expressed genes (DEGs) of the WRKYs and analyzed the transcriptome data of 'Starkrimson' pears and its green mutant at 40, 55, and 85 day after full bloom (DAFB). By analyzing the transcript abundance of 66 DEGs via a heat map, we found that Pbr000122.1, Pbr032698.1, Pbr013092.1, and Pbr026903.1 were more highly expressed in the three developmental stages of 'Starkrimson' fruits than in the green-skinned fruits (Fig. 1a). Furthermore, a phylogenetic tree was constructed using the neighbor-joining method and bootstrap analysis 


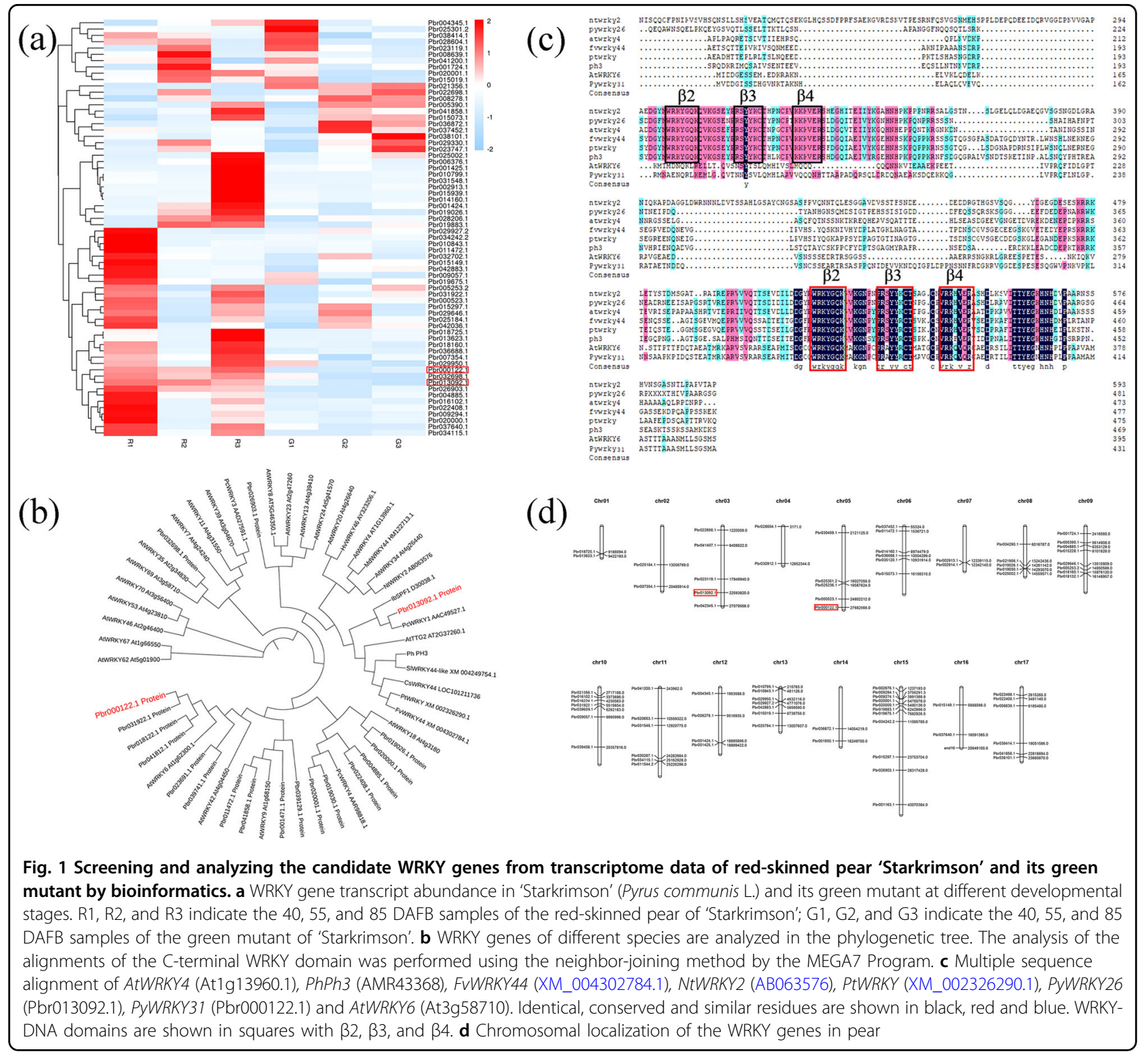

(1000 replicates) and MEGA7 software. The results indicated that the gene Pbr013092.1 (named PyWRKY26) has the most similar predicted protein sequences with homologous genes among all anthocyanin-related genes by a detailed phylogenetic analysis, which included the TFs AtWRKY4, FvWRKY44, NtWRKY2, PhPH3, PtWRKY1, and Pbr000122.1 (named PyWRKY31), which have high homology with AtWRKY6 (Fig. 1b). In this phylogenetic analysis, other PyWRKY sequences are part of different clusters and groups, further indicating the gene function of PyWRKY26 and PyWRKY31. Furthermore, analysis of the derived polypeptide alignment of PyWRKY26, PyWRKY31 and other genes involved in anthocyanin biosynthesis, such as WRKY TFs, revealed the presence of WRKY motifs in all these genes (Fig. 1c).
Moreover, the members of the WRKY gene family were widely distributed on seventeen chromosomes in pear (Fig. 1d). As shown in Fig. 1d, most WRKYs were closely located to each other. This finding might indicate that tandem duplication events occurred in the WRKY gene family. The genes PyWRKY26 (Pbr13092.1) and PyWRKY31 (Pbr000122.1) located at Chr 3 and Chr 5 were explored in this study.

\section{Evaluating the correlation of PyWRKY26/PyWRKY31 with anthocyanin accumulation and other factors regulating anthocyanin biosynthesis in pears}

To confirm the correlation of PyWRKY26/PyWRKY31 with anthocyanin and anthocyanin-related TFs, we determined the anthocyanin contents in red-skinned 


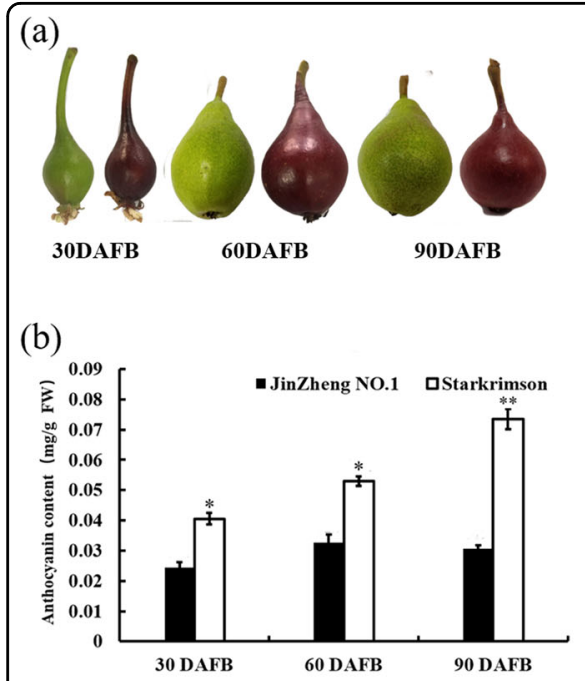

(c)
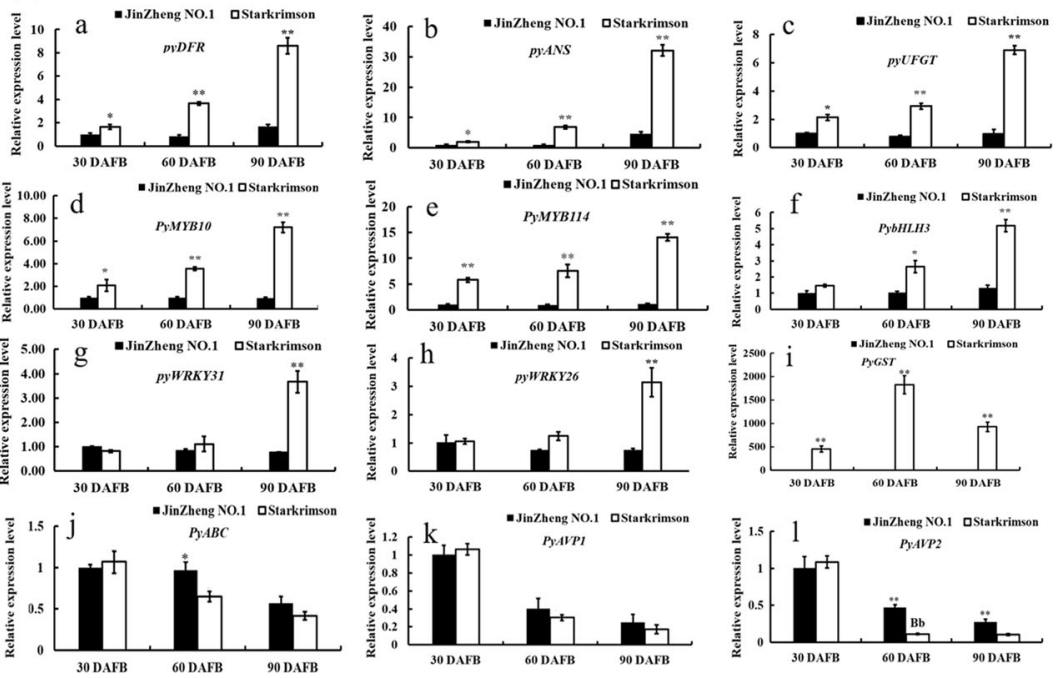

Fig. 2 Evaluating the correlation of the candidate WRKY genes with anthocyanin biosynthesis and transport-related genes in 'JinZheng No. 1' and 'Starkrimson' pear cultivars. a The appearance of the 'JinZheng No. 1' and 'Starkrimson' pears at 30, 60, and 90 DAFB. b Determination of the anthocyanin contents in the 'Starkrimson' and 'JinZheng No. 1' pears at 30, 60, and 90 DAFB. c The expression level was analyzed by RT-qPCR for anthocyanin metabolic structural and regulatory genes. Significant differences are indicated at the level of $P<0.05$ with lowercase letters. Highly significant differences are shown with uppercase letters $(P<0.01)$. Error bars are shown with the three biological replicates

'Starkrimson' and green-skinned 'Jinzheng No. 1' pears at different developmental stages. The results showed that the anthocyanin contents increased with fruit development in the 'Starkrimson' pear and were higher than those of the 'Jinzheng No. I' pear (Fig. 2a). Furthermore, the expression levels of PyDFR, PyANS, PyUFGT, PyMYB10, PyMYB114, PybHLH3, PyWRKY26, PyWRKY31, and PyGST were largely higher in the 'Starkrimson' pear than in the 'Jinzheng No. 1' pear (except PyABC transporter, $P y A V P 1$ and PyAVP2) (Fig. 2b, a-h). There was an obvious positive correlation between the anthocyanin contents and the anthocyanin biosynthesis structural genes and TFs, such as PyUFGT, PyGST, PyWRKY26, PyWRKY31, PyMYB114, and PybHLH3, and a negative correlation between the anthocyanin contents and $P y A B C$ transporter, PyAVP1 and PyAVP2 (Fig. S1).

\section{Heterologous expression of PyWRKY26/PyWRKY31 and other TFs induces anthocyanin accumulation in tobacco leaves}

To verify the function of PyWRKY31 and PyWRKY26 in anthocyanin synthesis, we transiently transformed these genes into tobacco leaves. As shown in Fig. 3a, the empty vector pSAK277 was injected as a negative control, pigmentation was not observed when PyWRKY31 or PyWRKY26 alone was transformed with PyMYB114, and little pigmentation could be observed after cotransforming PyMYB114 or PyMYB10 with PybHLH3. The pigmentation was largely enhanced when PyWRKY26 or PyWRKY31 was cotransformed with PyMYB114, PyMYB10, and PybHLH3.
Moreover, the anthocyanin contents in the tobacco leaves were analyzed by a colorimeter, and the changes in the $L^{*}, a^{*}$, and $b^{*}$ values were consistent with our expectations (Fig. 3b). When PyWRKY26/PyWRKY31 was involved in cotransformation, the anthocyanin content in tobacco was significantly higher than that when only PyMYB114, PyMYB10, and PybHLH3 were cotransformed (Fig. $3 \mathrm{c})(P<0.01)$. The above results indicated that cotransformation of PyWRKY26/ PyWRKY31 with PyMYB10, PyMYB114, and PybHLH3 could significantly promote anthocyanin synthesis.

\section{Overexpression of PyWRKY26/PyWRKY31 with other related TFs results in anthocyanin accumulation in strawberry receptacles}

To further identify the function of PyWRKY26 and PyWRKY31 in anthocyanin synthesis, we performed transient transformation of strawberry receptacles. As shown in Fig. 4a, no pigmentation was observed in the empty vector pSAK277-transformed receptacles. The pattern of change was similar to that of tobacco leaves; PyMYB114+ PyWRKY31 or PyMYB114+ PyWRKY26 were cotransformed, and no pigmentation was observed. Some pigmentation could be observed when PyMYB10 + PybHLH3 or PyMYB114 + PybHLH3 were cotransformed. A deeper color change could be observed when PyMYB114, PyMYB10, and PybHLH3 were coinjected. Meanwhile, the accumulation of anthocyanin was significantly higher when three TFs, PyMYB114, PyMYB10, and PybHLH3, were coinjected with PyWRKY26/PyWRKY31. In addition, the changes in the $L^{*}, a^{*}$, and $b^{*}$ values were influenced by the 

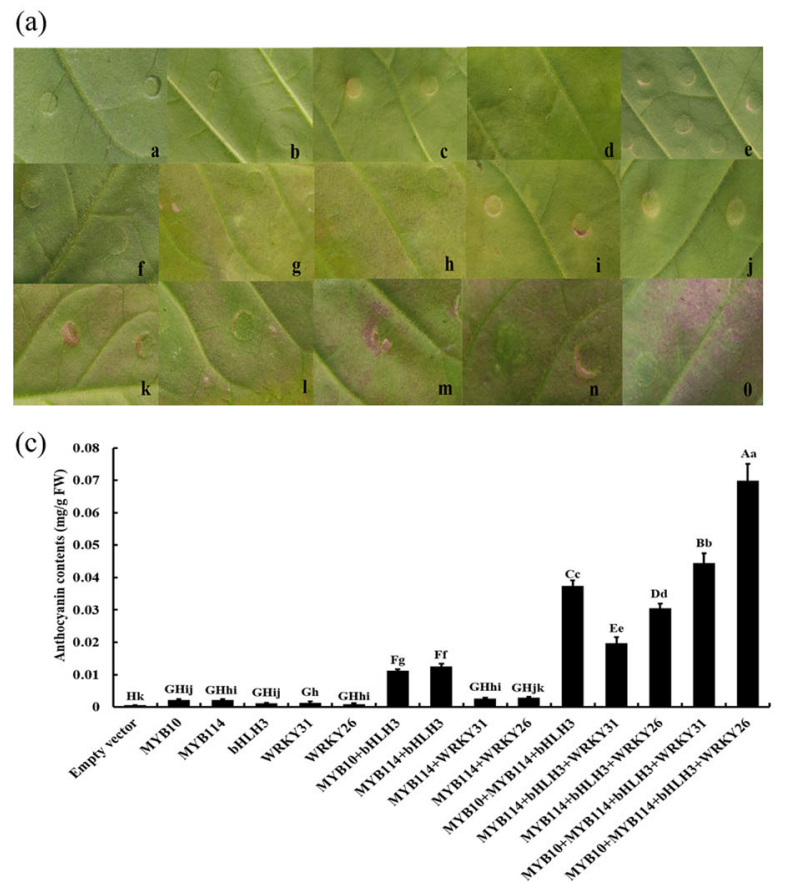

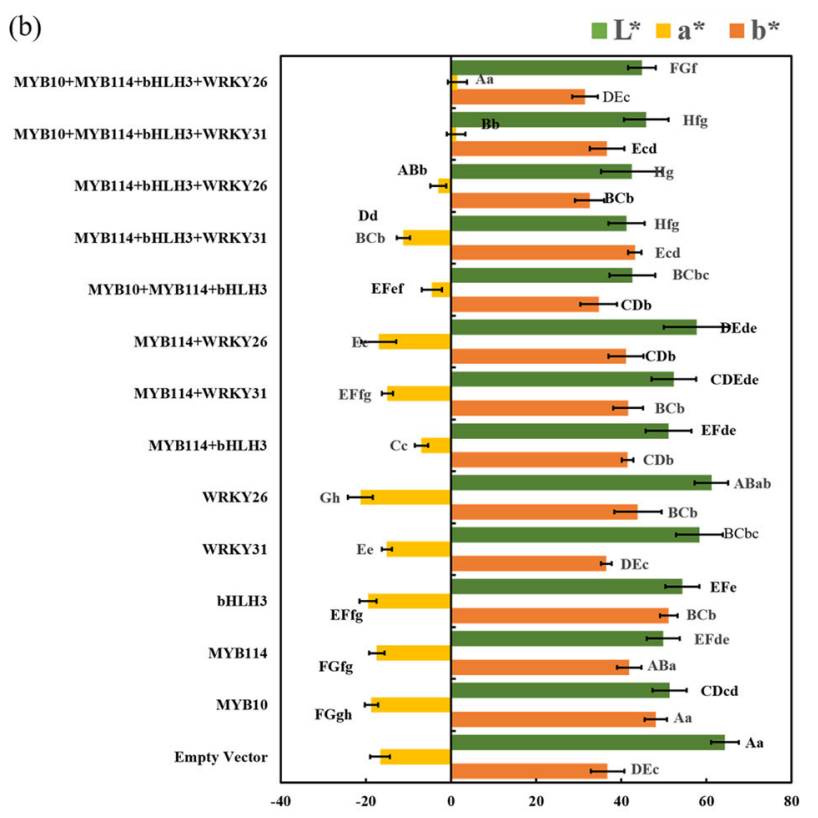

Fig. 3 Cotransformation of PyWRKY31/PyWRKY26 with its partners induced anthocyanin biosynthesis, as shown by transient expression assays in tobacco leaves. a The appearance of tobacco leaves $5 \mathrm{~d}$ after injection: a, pSAK277; b, PyMYB10; c, PyMYB114; d, PybHLH3; e, PyWRKY31; f, PyWRKY26; g, PYMYB10 + PybHLH3; h, PyMYB114 + PybHLH3; i, PyMYB114 + PyWRKY31; j, PyMYB114 + PyWRKY26; k, PyMYB10 + PyMYB114 + PybHLH3; l, PyMYB114 + PybHLH3 + PyWRKY31; m, PyMYB114 + PybHLH3 + PyWRKY26; n, PyMYB10 + PyMYB114 + PybHLH3 + PyWRKY31; o, PyMYB10 + PyMYB114 + PybHLH3 + PyWRKY26. $\mathbf{b}$ Differences in color are shown with the values of $\mathrm{L}^{*}, \mathrm{a}^{*}$, and $\mathrm{b}^{*}$. Significant differences are indicated at the level of $P<0.05$ with lowercase letters. Highly significant differences are shown at the level of $P<0.01$ with uppercase letters. Error bars are shown for six biological replicates. c Determination of the total anthocyanin contents in tobacco leaves with induced coloration

color change of the strawberry receptacles (Fig. 4b). When PyWRKY26 was coinjected with PyMYB114, PyMYB10, and PybHLH3, the total anthocyanin content in strawberry was significantly higher than that with only the cotransformation of PyMYB114, PyMYB10, and PybHLH3 (Fig. 4c) $(P<$ 0.01). Meanwhile, when PyWRKY31 was coinjected with PyMYB114, PyMYB10, and PybHLH3, the similar results for the anthocyanin contents were obtained. Overall, cotransformation of PyWRKY26/PyWRKY31 with PyMYB10, $P y M Y B 114$, and PybHLH3 can significantly enhance the anthocyanin biosynthesis in strawberry receptacles.

RT-qPCR analysis of the expression levels of anthocyanin biosynthesis- and vacuolar transport-related genes in strawberry receptacles

To investigate the mechanism of PyWRKY26/ PyWRKY31 in regulating anthocyanin metabolism, we evaluated six structural genes of anthocyanin biosynthesis and transport in strawberry receptacles by RT-qPCR analysis. FvDFR, FvANS, and FvUFGT have been previously reported to control anthocyanin biosynthesis. The other three genes, FvGST, FvABC transporter, and FvAVPs, were identified as key genes involved in anthocyanin transport. As shown in Fig. 4d-f, cotransformation of PyWRKY26/PyWRKY31 with PyMYB10, PyMYB114, and $P y b H L H 3$ significantly enhanced the expression levels of the FvDFR, FvANS, FvUFGT, and FvGST genes. Meanwhile, PyWRKY26 had a stronger upregulation than PyWRKY31. Compared with pSAK277 alone, the FvAVP and the $F v A B C$ transporter genes were significantly repressed when four TFs were cotransformed into the strawberry receptacles (Fig. 4h-i). RT-qPCR analysis suggested that cotransformation of four TFs, PyMYB10, PyMYB114, PybHLH3, and PyWRKY26/PyWRKY31, enhanced the anthocyanin synthesis and transport by upregulating the expression of the FvDFR, FvANS, FvUFGT, and FvGST genes and downregulating the expression of the FvAVPs and FvABC transporter genes in the strawberry receptacle.

\section{Validation of the interaction of the transcriptional regulatory complex by a dual-luciferase reporter system}

A dual-luciferase reporter assay was used to verify the interaction of our candidate TFs with the structural genes, including PyDFR, PyANS, PyUFGT, PyGST, PyABC transporter, and PyAVP1/2 in Nicotiana tabacum. The results showed that PyMYB10 and PyMYB114 cotransformed with $P y b H L H 3$ could induce the activity of these 


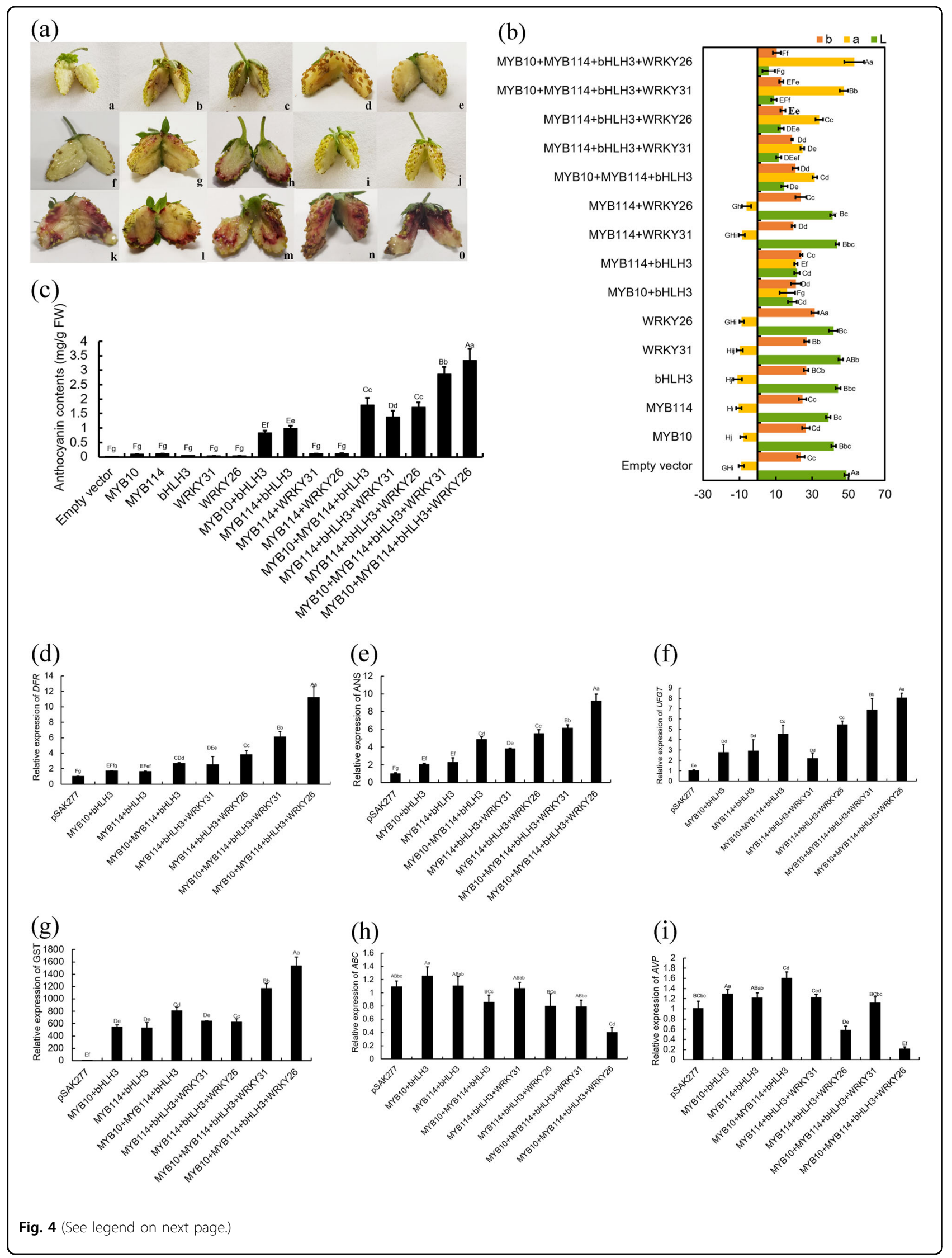


(see figure on previous page)

Fig. 4 The functional analysis of PyWRKY31/PyWRKY26 cotransformed with its partners, which resulted in anthocyanin biosynthesis, as shown by transient expression assays in strawberry receptacles. a The appearance of the strawberry receptacles $5 \mathrm{~d}$ after infiltration: a, pSAK277; b, PyMYB10; c, PyMYB114; d, PybHLH3; e, PyWRKY31; f, PyWRKY26; g, PyMYB10 + PybHLH3; h, PyMYB114 + PybHLH3; i, PyMYB114 + PyWRKY31; j, PyMYB114 + PyWRKY26; k, PyMYB10 + PyMYB114 + PybHLH3; l, PyMYB114 + PybHLH3 + PyWRKY31; m, PyMYB114 + PybHLH3 + PyWRKY26; n, PyMYB10 + PYMYB114 + PybHLH3 + PyWRKY31; O, PYMYB10 + PyMYB114 + PybHLH3 + PyWRKY26. b Differences in color are shown with the values of $L^{*}, a^{*}$ and $b^{*}$. Significant differences are indicated at the level of $P<0.05$ with lowercase letters, and highly significant differences are shown at the level of $P<$ 0.01 with uppercase letters. Error bars are based on six biological replicates. $\mathbf{c}$ Determination of the total anthocyanin contents. Error bars are based on three biological replicates. d-i RT-qPCR analysis of the expression levels of the genes FvDFR, FvANS FvUFGT, FvGST, FvABC transporter and FvAVP. Significant differences are indicated at the level of $P<0.05$ with lowercase letters. Highly significant differences are indicated with uppercase letters at $P<0.01$. Error bars show the SEs of the means $(n=3)$

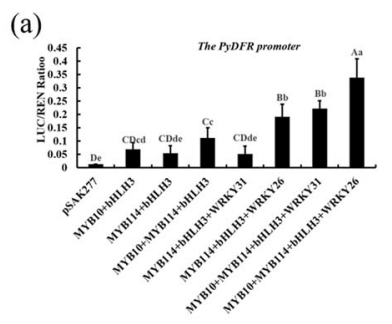

(d)

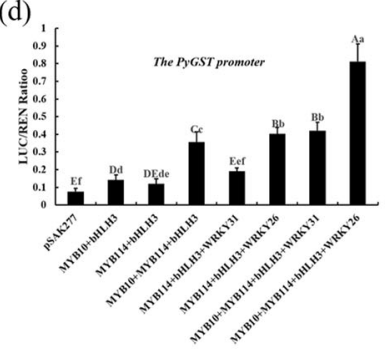

(e) (b)

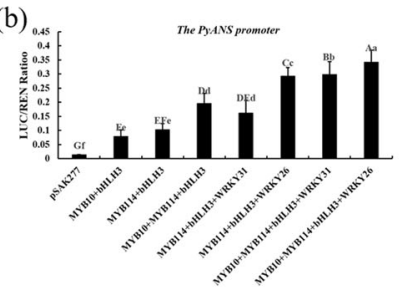

(c)

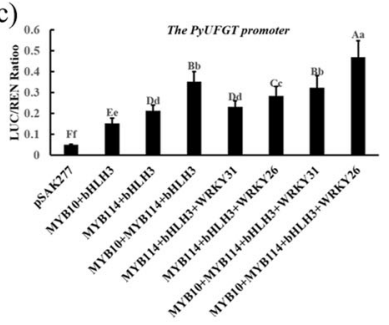

(f) (g)

Fig. 5 The transactivate activity of TFs PyMYB10, PyMYB114, PybHLH3, and PyWRKYs for anthocyanin biosynthesis and transport related genes was verified by a dual-luciferase reporter assay. The dual-luciferase reporter assay verified that PyMYB10, PyMYB114, PybHLH3 and PyWRKY26/PyWRKY31 cotransformation induces the activity of the PyDFR (a), PyANS (b), PyUFGT (c), PyGST (d), PyABC transporter (e) and PyAVP1/2 (f, $\mathbf{g})$ promoters. The LUC/REN value was used to express promoter activity. Error bars represent the SEs for three replicate reactions

promoters, and the additional TF PyWRKY26/PyWRKY31 could significantly enhance the transactivation activity of the PyDFR, PyANS, PyUFGT, and PyGST promoters (Fig. 5a-d). Moreover, compared with that of pSAK277, the transactivation of the PyABC transporter and $P y A V P 1 / 2$ promoters was not obviously activated when PyMYB114, PyMYB10, PybHLH3, and PyWRKY26/ PyWRKY31 were cotransformed (Fig. $5 \mathrm{e}-\mathrm{g}$ ). These results indicated that PyWRKY26/PyWRKY31 coregulates anthocyanin accumulation through coordination with the PyMYB10 + PyMYB114 + PybHLH3 complex.

\section{Activation activity of PyWRKY26 and PybHLH3 on the PyMYB114 promoter was verified}

To further explore the relationship of the TFs PyMYB114, PybHLH3, and PyWRKY26/PyWRKY31, we cloned the upstream $2 \mathrm{~kb}$ promoter of $P y M Y B 114$ and analyzed it by a dual-luciferase reporter assay in tobacco leaves. As shown in Fig. 6a, cotransformation with
PyWRKY26 and PybHLH3 had a stronger activation effect on the promoter sequences of PyMYB114 than other factors, including PyWRKY31. The binding of PybHLH3 and PyWRKY26 to the PyMYB114 promoter was further verified by yeast one-hybrid technology. Promoter structure analysis revealed that multiple cis-regulatory elements were predicted by PlantCARE (Fig. 6b). The promoter segment baits were fused to the prey vectors pGADT7-PybHLH3 and pGADT7-PyWRKY26 and introduced into the Y1HGold yeast strain, and the results suggested that PyWRKY26 could bind to the S2 fragments. However, we did not detect a direct association between PybHLH3 and the promoter of PyMYB114, although several binding sites were located in different individual promoter regions (Fig. 6c).

\section{Verification of the interaction of PybHLH3 with PyWRKY26}

For further identification of the interaction of $\mathrm{PybHLH} 3$ with PyWRKY26, a tobacco-based firefly luciferase 


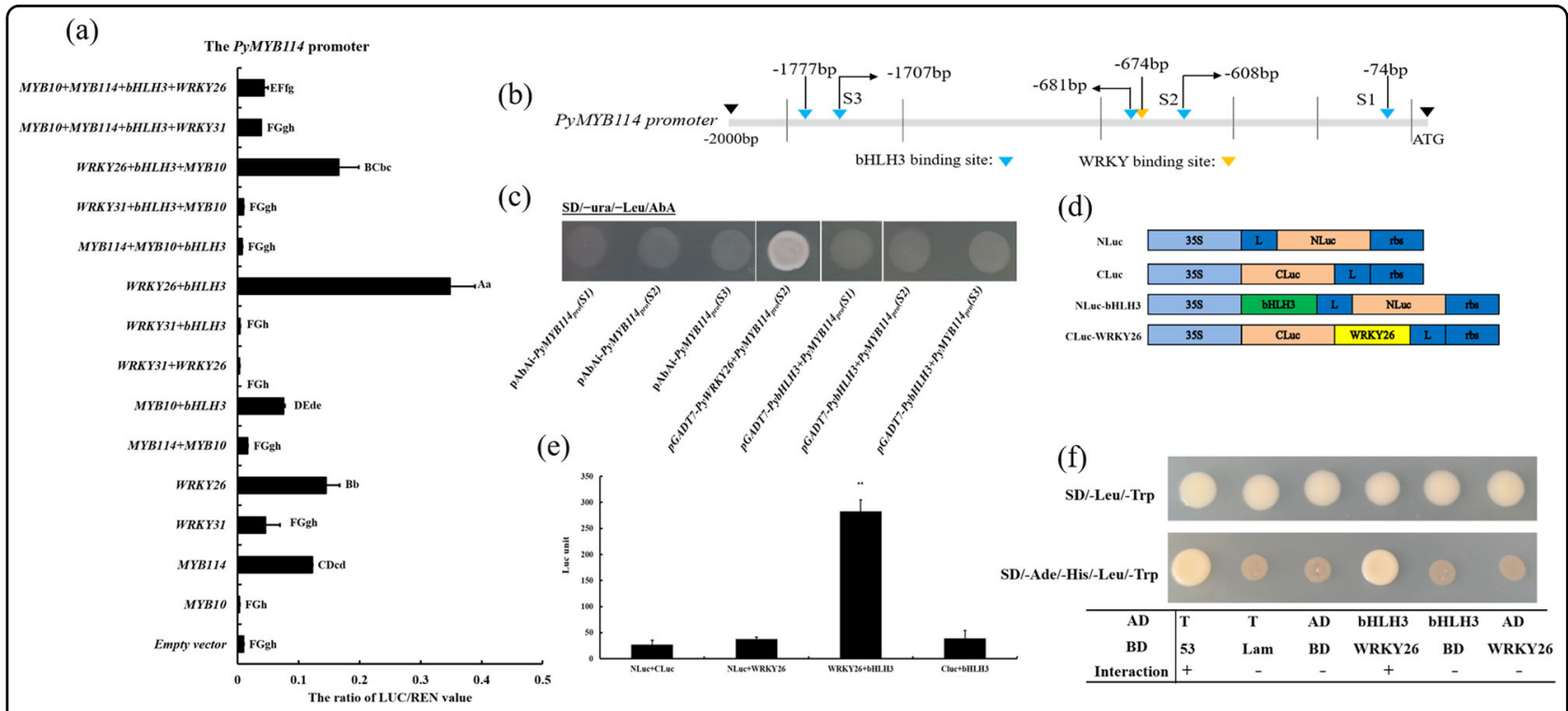

Fig. 6 Verifying the regulatory pattern of TFs PyMYB114, PybHLH3, and PyWRKY26 in vivo. a The dual-luciferase reporter assay verifying PybHLH3 and PyWRKY26 cotransactivation activity. b A schematic of the PyMYB114 promoter. The bar segments were analyzed by using the PLACE and PlantCare databases and are divided by solid lines (S1-S3). c Yeast one-hybrid assays between PybHLH3/PyWRKY26 and the PyMYB114 promoter. Positive colonies indicated strong specific interactions with the PyMYB114 promoter segments. The colony name indicates the bait segment, and prey vectors were used. $\mathbf{d}$ Model of the NLUC, CLuc and NLuc/CLuc constructs. e Firefly luciferase complementation assays in young tobacco leaves. Error bars are shown with six biological replicates. ${ }^{*} P<0.01$. f Verification of the interaction of PyWRKY26 and PybHLH3 in vivo

complementation assay was performed. PybHLH3 was inserted into the $\mathrm{N}$-terminal region of the firefly luciferase (NLuc), whereas PyWRKY26 was linked to the C-terminal region of the firefly luciferase (CLuc) (Fig. 6d). Coexpression of the NLuc-PybHLH3 and CLuc-PyWRKY26 constructs showed the strongest capability to rescue intense luciferase enzyme activity $(P<0.01)$. Nevertheless, for the control constructs, including NLuc-PybHLH3 with $\mathrm{C}$ and CLuc-PyWRKY26 with Nluc, no obvious luciferase enzyme activity was observed (Fig. 6e).

Then, the interaction of PyWRKY26 with PybHLH3 was further verified by $\mathrm{Y} 2 \mathrm{H}$ assays. The full-length coding sequences of PyWRKY26 and PybHLH3 were inserted separately into pGBKT7 and pGADT7. The results indicated that cotransformed PyWRKY26 and PybHLH3 in AH109 yeast cells resulted in healthy growth on the medium (Fig. 6f). Above all, the results suggested that $P y W R K Y 26$ interacts with PybHLH3 in vivo.

\section{Discussion}

Anthocyanin, a common secondary metabolite in plants that exhibits antioxidant properties, gives plants a red or purple color. At present, an extensive body of literature has clearly demonstrated that the anthocyanin biosynthetic pathway is regulated at the transcriptional level by an MYB-bHLH-WD40 (MBW) regulatory complex in plants, and MYB in particular plays a vital role in anthocyanin synthesis. Compared with MYB, the bHLH TF has a significantly lower functional specificity, similar to AtEGL3, AtGL3, and AtTT8, and the three bHLH TFs have functional redundancy in the regulation of anthocyanin biosynthesis and trichome development ${ }^{15-17}$. In addition, WD40 plays an important role in enhancing the stability of the MBW complex, and the interaction between MYB and bHLH TFs is a prerequisite for specific recognition of DNA sequences ${ }^{19,34}$. In peach, the expression of PpMYB10.1 was activated with partner TF helix-loop-helix proteins and PpNAC1 and coregulated anthocyanin biosynthesis $^{35}$. In pear, Yao et al. showed that PyMYB114/ $P y M Y B 10$, which interacts with PybHLH3 in tobacco leaves, strawberry receptacles and pears, could promote the biosynthesis of anthocyanins. Moreover, PyERF3 promoted anthocyanin biosynthesis by coexpression with $P y M Y B 114$ and $P y b H L H 3^{18}$. In this study, we also demonstrated that PyWRKY26/PyWRKY31 could be coexpressed with PyMYB114 and PybHLH3, resulting in anthocyanin biosynthesis in tobacco leaves and strawberry receptacles (Figs 3, 4). Thus, the regulatory networks of color formation in red-skinned pears are highly complex, and whether and how the interaction of PyWRKY26/PyWRKY31 with PyERF3 regulates anthocyanin biosynthesis still need further study.

The important TF regulatory family WRKY plays important roles in various plant processes, such as plant growth and metabolism and senescence ${ }^{35-37}$. WRKYs were shown to exert their biological functions and regulate metabolite biosynthesis, especially that of secondary metabolites, through physical interactions with different proteins $^{38}$. Verweij et al. reported that PH3, which 
encodes a WRKY protein of petunia, is a target gene of the AN11-AN1-PH4 complex, could bind to AN11 and is required together with the AN11-AN1-PH4 complex for the transcription of PH5 (which encodes an MYB protein), which regulates hair development, tannin accumulation, and mucilage production in Arabidopsis ${ }^{31}$. In our study, PyWRKY26 largely enhanced anthocyanin accumulation after transient cotransformation with PyMYB114 and PybHLH3 compared with the two cofactors PyMYB114+ PybHLH3 (Figs. 3 and 4). This evidence proved that WRKY needs to interact with other partner factors to carry out its biological functions. The regulatory complex was consistent with Verweij et al. ${ }^{31}$, although PyWRKY26 could regulate anthocyanin accumulation, while PH3 regulates tannin accumulation and mucilage production. Thus, TF WRKYs are involved in the formation of different secondary metabolites. In addition, we further explored the target genes of PyWRKY26 and PyWRKY31, and cotransformation with PyWRKY26 and PybHLH3 had a stronger activation effect on the promoter sequences of PyMYB114 than other factors. PyWRKY26 directly activated the promoter sequences of $P y M Y B 114$ (Fig. 6b, c), but PyWRKY31 could not bind to the PyMYB114 promoter (Fig. 6a). Further, the interaction of PyWRKY26 with PybHLH3 was proven by firefly luciferase complementation and $\mathrm{Y} 2 \mathrm{H}$ assays (Fig. $6 \mathrm{~d}-\mathrm{f}$ ). Therefore, PyWRKY26 and PyWRKY31 could regulate color formation, but the downstream target genes were different.

Generally, TFs forming regulatory complexes regulate anthocyanin accumulation by a series of anthocyanin biosynthesis- and transport-related structural genes. In this study, the expression levels of PyDFR, PyANS, PyUFGT, and PyGST in red-skinned pear were generally higher than those in green-skinned pear, and the expression patterns of $A B C$ transporters and $A V P s$ were opposite (Fig. 2). Furthermore, cotransformation of PyWRKY26 with its partners in strawberry receptacles showed that the expression of FvDFR, FvANS, FvUFGT, and $F v G S T$ was upregulated and $F v A B C$ transporter and FvAVPs were downregulated (Fig. 4). The dual-luciferase reporting system also demonstrated a similar conclusion (Fig. 5). It has been widely reported that DFR, ANS, and UFGT catalyze anthocyanin biosynthesis. GST has been shown to be involved in anthocyanin transport in strawberry $^{39}$. Recently, the ATP-binding cassette transporter $A t A B C C 2$ was reported to be involved in the vacuolar transport of anthocyanins and other flavonoids in Arabidopsis $^{40}$. In a previous study, GST acted as a carrier of anthocyanins and mobilized anthocyanins from the cytoplasm to the tonoplast by the $\mathrm{ABC}$ transmembrane transporter ${ }^{8,9}$. Many secondary transporters and channels, such as malate transporter, MATE-type transporters and $\mathrm{ABC}$ transporters, exist on the tonoplast, and the activities of these transporters are directly or indirectly dependent on the proton gradient generated by V-ATPase and VPpase ${ }^{10,11,41-43}$. The $\mathrm{H}^{+}$-pumping activities of vacuolar $\mathrm{H}^{+}$-ATPase (VHA) and pyrophosphatase (VHP) have an extremely important role in the transport of anthocyanins, malate and other metabolites ${ }^{44}$. These reports were consistent with the findings of our study showing involvement in the vacuolar transport of anthocyanins, but previous reports did not present evidence of the relationship between transport-related genes and the transcription regulatory complex. Moreover, we confirmed that the regulatory mode of the PyABC transporter and $P y A V P S$ is different from that of $P y G S T$, and we speculate that there is a difference in the timing between the anthocyanin synthetic process and transport process in plant cells. When the synthetic process was significantly activated, which results in anthocyanin accumulation, PyGST participates in transport over time, but the PyAVP and $P y A B C$ transporter genes are not activated. Anthocyanin biosynthesis and anthocyanin transport may be independent processes, although they are regulated by the same transcription complex; furthermore, they are not expressed simultaneously. The molecular mechanism by which transporter genes participate in anthocyanin transport still requires further research.

\section{Conclusion}

In this study, the TFs PyWRKY31 and PyWRKY26 with their partners PyMYB10, PyMYB114, and PybHLH3 were cotransformed into tobacco leaves and strawberry receptacles and resulted in increased anthocyanin contents. Furthermore, we confirmed that this cotransformation activated the activity of PyDFR, PyANS, and PyUFGT in anthocyanin biosynthesis and PyGST in anthocyanin transport instead of the $P y A B C$ transporter and $P y A V P s$. Moreover, firefly luciferase reporter assays and yeast expression assays showed that the interaction of PyWRKY26 and PybHLH3 could cotarget the PyMYB114 promoter and that $P y W R K Y 26$ directly activates the promoter sequences of $P Y M Y B 114$, which resulted in anthocyanin accumulation in red-skinned pear. This research provides novel insight into the regulatory network of anthocyanin accumulation and contributes to improving the appearance quality of red-skinned pears.

\section{Materials and methods \\ Plant materials}

The green-skinned pear 'JinZheng No. 1' and redskinned pear 'Starkrimson' used in this study were collected from the orchard of the Institute of Pomology in Shandong Province during the 2018 growing season. Tobacco (N. tabacum) and diploid strawberry (Fragaria vesca, called 'Yellow Wonder' 5AF7) were used in this study for the transient transformation experiments, dualluciferase reporter system assays and firefly luciferase 
complementation assays, and they were cultivated in an intelligent incubator with a $16 \mathrm{~h}$ photoperiod and a $21^{\circ} \mathrm{C} /$ $17^{\circ} \mathrm{C}$ day/night temperature. Young tobacco leaves and strawberry receptacles $\sim 2$ weeks after flowering were infiltrated for the experiment, and observations at 4-6 d after injection were performed. The tobacco leaves and strawberry receptacles were collected and chopped, frozen with liquid nitrogen and stored at $-80^{\circ} \mathrm{C}$.

\section{Extraction and determination of the anthocyanins in tobacco leaves and strawberry}

Anthocyanins were extracted according to the method of Yang et al. ${ }^{33}$. Approximately $0.2 \mathrm{~g}$ of the fruit skin of 'JinZheng No. 1' and 'Starkrimson', tobacco leaves or strawberry receptacles stored at $-80{ }^{\circ} \mathrm{C}$ were ground to powder in liquid nitrogen and then homogenized with $1 \mathrm{ml}$ of cold methanol containing $0.1 \% \mathrm{HCl}$ at $4{ }^{\circ} \mathrm{C}$ for $24 \mathrm{~h}$, and the homogenate was allowed to incubate in the dark at $4{ }^{\circ} \mathrm{C}$ for $24 \mathrm{~h}$. Then, the supernatant was collected by centrifugation at $12,000 \mathrm{rpm}$ for $20 \mathrm{~min}$. The absorbance of the anthocyanins was measured at wavelengths of 530, 620, and $650 \mathrm{~nm}$ using a Multiskan Spectrum (Thermo Scientific Multiskan GO 1510, Finland). There were three biological replicates per sample, and the total content of anthocyanin per sample fresh weight was calculated according to the following formula: $\mathrm{OD}=\left(\mathrm{A}_{530}-\right.$ $\left.\mathrm{A}_{620}\right)-0.1 \times\left(\mathrm{A}_{650}-\mathrm{A}_{620}\right)$.

\section{Total RNA extraction and gene transcript abundance analysis by RT-qPCR}

Fruit skin of 'JinZheng No. 1' and 'Starkrimson' and strawberry receptacles were ground into fine powder in liquid nitrogen. Total RNA of the powder samples was extracted in an ice bath. First strand cDNA synthesis was performed using Prime Script RT Master Mix (DRR036A, TaKaRa, China). RT-qPCR was conducted using SYBR Premix Ex Taq ${ }^{\text {TM }}$ II (DRR081A, TaKaRa, China) in a $10 \mu \mathrm{l}$ volume. The amplification program was as follows: one cycle of $30 \mathrm{~s}$ at $95^{\circ} \mathrm{C}$, followed by 40 cycles of $15 \mathrm{~s}$ at $95^{\circ} \mathrm{C}$ and $30 \mathrm{~s}$ at $60^{\circ} \mathrm{C}$; a strawberry housekeeping gene (gene11892) was used as an internal control. The relative expression level of the genes was calculated using the $2^{(-\Delta \Delta \mathrm{Ct})}$ method. All analyses and error bars were determined using three biological replicates. The primer sequences for RT-qPCR are listed in Table S1.

\section{Genes cloning and overexpression vector construction}

The gene sequences of PyWRKY26 and PyWRKY31 cloned from the cDNA of the red-skinned pear cultivar 'Starkrimson' were used in the following experiment. PCR amplification was conducted using TransStart FastPfu DNA Polymerase (AP221-01, Transgen, China) and the primer sequences listed in Table S2. The PyWRKY26 and PyWRKY31 genes were inserted into the pSAK277 vector under the control of the $35 \mathrm{~S}$ promoter with EcoRI and $X \mathrm{XoI}^{45}$. The integrated constructs were transformed into the Agrobacterium tumefaciens strain GV3101 using the chemical method, and the cells were incubated at $28^{\circ} \mathrm{C}$ for $2 \mathrm{~d}$. A description of the infecting is in Yao et al. ${ }^{18}$. The specific method of infiltration experiments was described by Voinnet et al. ${ }^{46}$. Tobacco leaves and strawberry receptacles were collected for anthocyanin measurement and RNA extraction at 5-7 d after infiltration. Empty vector infiltrations (pSAK277) were used as negative controls.

\section{Dual-luciferase reporter system assays}

For the dual-luciferase reporter assay, the upstream promoter sequences of PyMYB114 (2.0 kb), PyAVP1/2 (1.8 kb), PyDFR $(2.0 \mathrm{~kb}), \quad$ PyANS $(2.0 \mathrm{~kb}), \quad$ PyUFGT (1.8 kb), PyABC transporter $(2.0 \mathrm{~kb})$, and $P y G S T$ (2.0 kb) were cloned and inserted into the pGreen II 0800-LUC vector with the primers listed in Table S2. The recombinant plasmids were transformed into the Agrobacterium strain GV3101 (PM90) with the pSoup helper plasmid. The TFs PyWRKY26 and PyWRKY31, PyMYB114, $P y M Y B 10$, and PybHLH3 were mixed with the promoter sequences (the ratio $1: 9, \mathrm{v} / \mathrm{v}$ ) and then injected into young tobacco leaves for transient cotransformation expression analysis ${ }^{18}$. According to the manufacturer's instructions, the ratio of transactivation activities of firefly luciferase and renilla luciferase was tested by the Dual-Luciferase Reporter Assay System (E1910, Promega, USA).

\section{Firefly luciferase complementation assay}

Firefly luciferase complementation assays were performed according to the method of Chen et al ${ }^{47}$. Gene sequences of PybHLH3 (with no stop codon) were amplified and linked with pCAMBIA1300-NLuc, and the coding sequences of PyWRKY26 were cloned and linked with the pCAMBIA1300-CLuc vector. The primer sequences are listed in Table S2. Then, the cells were transformed into Agrobacterium GV3101 and cultured using a selection medium containing kanamycin. The cells were grown to $\mathrm{OD}_{600} 0.6$, and then, bHLH3-NLuc and PyWRKY26-CLuc were mixed 1:1 by volume and infiltrated into the tobacco leaves. Leaf disks (exactly $2 \mathrm{~cm}$ in diameter) were punched adjacent to the infiltration site, and the firefly luciferase activity was determined by a Steady-Glo ${ }^{\circ}$ Luciferase Assay System (E2510, Promega, USA).

\section{Yeast one-hybrid assay}

To identify transcriptional regulators of PyMYB114 by yeast one-hybrid assays, we used the $\sim 300$ bp promoter segments, corresponding to the $\mathrm{S} 1-\mathrm{S} 3$ sequences. The promoter fragments were inserted into the pAbAi vector, and the PybHLH3 and PyWRKY26 genes were cloned into 
the pGADT7 vector. In a preliminary filter, self-activation of the bait vectors was tested on $\mathrm{SD} /$-ura $+\mathrm{AbA}^{100}, \mathrm{SD} /$ ura $+\mathrm{AbA}^{200}$, and $\mathrm{SD} /$-ura $+\mathrm{AbA}^{400}$ plates; the prey vectors pGADT7-PybHLH3 and pGADT7-PyWRKY26 were tested on SD/-Leu plates. The promoter segment baits were fused to the prey vectors pGADT7-PybHLH3 and pGADT7-PyWRKY26 and introduced into the Y1HGold yeast strain and tested on $\mathrm{SD} /$-ura $+\mathrm{AbA}$ plates at $30^{\circ} \mathrm{C}$ for $3 \mathrm{~d}$. The primer sequences used for vector construction are listed in Supporting Information Table S1.

\section{Yeast two-hybrid assay}

According to the Matchmaker ${ }^{\oplus}$ Gold Yeast Two-Hybrid System (Clontech, HTTP:// www.clontech.com/), a Y2H assay was performed to test for protein interactions. The PyWRKY26 and PybHLH3 genes were inserted separately into pGBKT7 and pGADT7 and then cotransformed into the yeast strain AH109. The transformants were selected on SD/-Leu/-Trp medium and tested on SD/-Leu/-Trp/His/-Ade medium. Meanwhile, pGADT7-T and pGBKT7Lam or pGADT7-T and pGBKT7-53 were cotransformed as negative and positive controls.

\section{Statistical analysis}

All samples were assessed at least three times independently, and all data are represented as the mean $\pm \mathrm{SD}$. Statistical analysis was performed by Student's $t$-test and one-way ANOVA. Significance was indicated by asterisks $*(P<0.05)$ or $* *(P<0.01)$ or different letters.

\section{Acknowledgements}

This work was supported by the National Natural Science Foundation of China (31901993, 31970312, 31970200, 31670278, 31872078), the Natural Science Foundations of Anhui Province (1908085MC72), the Key Research and Development Program of Anhui Province (201904a06020031), and the Fundamental Research Funds for the Central Universities (JZ2018HGBZ0160, JZ2018HGTB0241). We thank Dr. Andrew C. Allan, Dr. Lin-Wang Kui and Dr. Richard Espley from The New Zealand Institute for Plant \& Food Research Limited, Auckland, New Zealand, for the dual vector pGreen II 0800-LUC.

\section{Author details}

${ }^{1}$ School of Food and Biological Engineering, Hefei University of Technology, 230009 Hefei, China. ${ }^{2}$ Centre of Pear Engineering Technology Research, State Key Laboratory of Crop Genetics and Germplasm Enhancement, Nanjing Agricultural University, 210095 Nanjing, China. ${ }^{3}$ Shandong Institute of Pomology, 271000 Taian, China. ${ }^{4}$ Anhui Province Key Laboratory of Functional Compound Seasoning, Anhui Qiangwang Seasoning Food Co., Ltd., 236500 Jieshou, China

\section{Author contributions}

C.L., G.F.Y., J.W., and H.Z. conceived and designed the experiments; C.L., G.F.Y., S.W.W., and H.Y.S. performed the experiments; Z.H. and H.L.Y. analyzed the data; C.L. and G.F.Y. wrote the paper; K.D.H., J.W., G.F.Y., and H.Z. interpreted the data and revised the manuscript.

\section{Conflict of interest}

The authors declare that they have no conflict of interest.

Supplementary Information accompanies this paper at (https://doi.org/ 10.1038/s41438-020-0254-z).
Received: 3 August 2019 Revised: 30 December 2019 Accepted: 15 January 2020

Published online: 15 March 2020

\section{References}

1. Wu, J. et al. Identification of differentially expressed genes related to coloration in red/green mutant pear (Pyrus communis L.). Tree Genet. Genomes 9, 75-83 (2013).

2. Winkel-Shirley, B. Flavonoid biosynthesis. A colorful model for genetics, biochemistry, cell biology, and biotechnology. Plant Physiol. 126, 485-493 (2001).

3. Butelli, E. et al. Enrichment of tomato fruit with health-promoting anthocyanins by expression of select transcription factors. Nat. Biotech. 26, 1301-1308 (2008).

4. Rasmussen, S. E., Frederiksen, H., Struntze Krogholm, K. \& Poulsen, L. Dietary proanthocyanidins: occurrence, dietary intake, bioavailability, and protection against cardiovascular disease. Mol. Nutr. Food Res. 49, 159-174 (2005).

5. Sun, L. L. et al. Composition and antioxidant activity of the anthocyanins of the fruit of Berberis heteropoda Schrenk. Molecules 19, 19078-19096 (2014).

6. Gomez, C. et al. In vivo grapevine anthocyanin transport involves vesiclemediated trafficking and the contribution of anthoMATE transporters and GST. Plant J. 67, 960-970 (2011).

7. Zhao, J. \& Dixon, R. A. MATE transporters facilitate vacuolar uptake of epicatechin 3'-O-glucoside for proanthocyanidin biosynthesis in Medicago truncatula and Arabidopsis. Plant Cell 21, 2323-2340 (2009).

8. Marrs, K. A., Alfenito, M. R., Lloyd, A. M. \& Walbot, V. A glutathione S-transferase involved in vacuolar transfer encoded by the maize gene Bronze-2. Nature 375, 397-400 (1995).

9. Sun, Y., Hong, H. \& Ji, R. Arabidopsis $T T 19$ functions as a carrier to transport anthocyanin from the cytosol to tonoplasts. Mol. Plant 5, 387-400 (2012).

10. Pasapula, V. et al. Expression of an Arabidopsis vacuolar $\mathrm{H}^{+}$-pyrophosphatase gene (AVP1) in cotton improves drought- and salt tolerance and increases fibre yield in the field conditions. Plant Biotech. J. 9, 88-99 (2011).

11. Li, J. S. et al. Arabidopsis $\mathrm{H}^{+}$-PPase AVP1 regulates auxin-mediated organ development. Science 310, 121-125 (2005).

12. Holton, T. A. \& Cornish, E. C. Genetics and biochemistry of anthocyanin biosynthesis. Plant Cell. 7, 1071-1083 (1995).

13. Feng, S., Wang, Y., Yang, S., Xu, Y. \& Chen, X. Anthocyanin biosynthesis in pears is regulated by a R2R3-MYB transcription factor PyMYB10. Planta 232, 245-255 (2010).

14. Zhang, $X$. et al. Differential gene expression analysis of yunnan red pear, Pyrus Pyrifolia, during fruit skin coloration. Plant Mol. Biol. Rep. 29, 305-314 (2011).

15. Espley, R. V. et al. Red coloration in apple fruit is due to the activity of the MYB transcription factor, MdMYB10. Plant J. 49, 414-427 (2007).

16. Li, Y. Y. et al. MdCOP1 ubiquitin E3 ligases interact with MdMYB1 to regulate light-induced anthocyanin biosynthesis and red fruit coloration in apple. Plant Physiol. 60, 1011-1022 (2012).

17. Umemura, H., Otagaki, S., Wada, M., Kondo, S. \& Matsumoto, S. Expression and functional analysis of a novel MYB gene, MdMYB110a, responsible for red flesh, not skin color in apple fruit. Planta 238, 65-76 (2013).

18. Yao, G. et al. Map-based cloning of the pear gene MYB114 identifies an interaction with other transcription factors to coordinately regulate fruit anthocyanin biosynthesis. Plant J. 92, 437-451 (2017).

19. Baudry, A. et al. TT2, TT8, and TTG1 synergistically specify the expression of BANYULS and proanthocyanidin biosynthesis in Arabidopsis thaliana. Plant J. 39, 366-380 (2004)

20. Liu, X. F. et al. The MrWD40-1 gene of Chinese Bayberry (Myrica rubra) interacts with MYB and bHLH to enhance anthocyanin accumulation. Plant Mol. Biol. Rep. 31, 1474-1484 (2013).

21. Jian, W. et al. SIMYB75, an MYB-type transcription factor, promotes anthocyanin accumulation and enhances volatile aroma production in tomato fruits. Hort. Res. 6, 1-15 (2019).

22. Zhou, H. et al. Molecular genetics of blood-fleshed peach reveals activation of anthocyanin biosynthesis by NAC transcription factors. Plant J. 82, 105-121 (2015).

23. Alessandra, A. et al. A grapevine TTG2-Like WRKY transcription factor is involved in regulating vacuolar transport and flavonoid biosynthesis. Front. Plant Sci. 7, 1979 (2017).

24. Peng, $X$. et al. Constitutive expression of rice WRKY30 gene increases the endogenous jasmonic acid accumulation, PR gene expression and resistance to fungal pathogens in rice. Planta 236, 1485-1498 (2012). 
25. Zentgraf, U., Laun, T. \& Miao, Y. The complex regulation of WRKY53, during leaf senescence of Arabidopsis thaliana. Eur. J. Cell Biol. 89, 133-137 (2010).

26. Chen, $L$. et al. The role of WRKY transcription factors in plant abiotic stresses. Biochim. Biophys. Acta 1819, 120-128 (2012)

27. $\mathrm{Xu}$, L. et al. Overexpression of GbWRKY1 positively regulates the Pi starvation response by alteration of auxin sensitivity in Arabidopsis. Plant Cell Rep. $\mathbf{3 1}$ 2177-2188 (2012).

28. Devaiah, B. N., Arthikeyan, A. S. \& Raghothama, K. G. WRKY75 transcription factor is a modulator of phosphate acquisition and root development in Arabidopsis. Plant Physiol. 143, 1789-1801 (2007).

29. Duan, S. et al. Functional characterization of a heterologously expressed Brassica napus WRKY41-1 transcription factor in regulating anthocyanin biosynthesis in Arabidopsis thaliana. Plant Sci. 268, 47-53 (2018).

30. Robatzek, S. \& Somssich, I. E. Targets of AtWRKY6 regulation during plant senescence and pathogen defense. Genes. Dev. 16, 1139-1149 (2002).

31. Verweij, C. W. et al. Functionally similar WRKY proteins regulate vacuolar acidification in petunia and hair development in Arabidopsis. Plant Cell $\mathbf{2 8}$ 786-803 (2016).

32. An, J. et al. MdWRKY40 promotes wounding-induced anthocyanin biosynthesis in association with MdMYB1 and undergoes MdBT2-mediated degradation. N. Phytol. 224, 380-395 (2019)

33. Yang, Y., Yao, G., Yue, W., Zhang, S. \& Wu, J. Transcriptome profiling reveals differential gene expression in proanthocyanidin biosynthesis associated with red/green skin color mutant of pear (Pyrus communis L.). Front. Plant Sci. 6, 795 (2015).

34. Hichri, I. et al. Recent advances in the transcriptional regulation of the flavonoid biosynthetic pathway. J. Exp. Bot. 62, 2465-2483 (2011).

35. Sperotto, R. A., Boff, T., Duarte, G. L. \& Fett, J. P. Increased senescenceassociated gene expression and lipid peroxidation induced by iron deficiency in rice roots. Plant Cell Rep. 27, 183-195 (2008).
36. Pandey, S. P. \& Somssich, I. E. The role of WRKY transcription factors in plant immunity. Plant Physiol. 150, 1648-1655 (2009).

37. Zhou, J. et al. Global genome expression analysis of rice in response to drought and high-salinity stresses in shoot, flag leaf, and panicle. Plant Mol Biol. 63, 591-608 (2007).

38. Rushton, P. J., Somssich, I. E., Ringler, P. \& Shen, Q. J. WRKY transcription factors. Trends Plant Sci. 15, 247-258 (2010).

39. Luo, H. et al. Reduced anthocyanins in petioles codes for a GST anthocyanin transporter that is essential for the foliage and fruit coloration in strawberry. J. Exp. Bot. 69, 2595-2608 (2018).

40. Behrens, C. E., Smith, K. E., lancu, C. V., Choe, J. Y. \& Dean, J. V. Transport of anthocyanins and other flavonoids by the Arabidopsis ATP-binding cassette transporter AtABCC2. Sci. Rep. 9, 437 (2019).

41. Hu, D. et al. The R2R3-MYB transcription factor MdMYB73 is involved in malate accumulation and vacuolar acidification in apple. Plant J. 91, 443-454 (2017)

42. Francisco, R. M. et al. ABCC1, an ATP binding cassette protein from grape berry, transports anthocyanidin 3-O-Glucosides. Plant Cell 25, 1840-1854 (2013).

43. Gomez, C. et al. Grapevine MATE-type proteins act as vacuolar $\mathrm{H}^{+}$-dependent acylated anthocyanin transporters. Plant Physiol. 150, 402-415 (2009).

44. Shiratake, K. \& Martinoia, E. Transporters in fruit vacuoles. Plant Biotech. 24 127-133 (2007)

45. Hellens, R. P. et al. Transient expression vectors for functional genomics, quantification of promoter activity and RNA silencing in plants. Plant Methods 1, 1-13 (2005).

46. Voinnet, O., Rivas, S., Mestre, P. \& Baulcombe, D. Retracted: an enhanced transient expression system in plants based on suppression of gene silencing by the p19 protein of tomato bushy stunt virus. Plant J. 33, 949-956 (2003).

47. Chen, $\mathrm{H}$. et al. Firefly luciferase complementation imaging assay for proteinprotein interactions in plants. Plant Physiol. 146, 368-376 (2008). 